\title{
STAPHYLOCOCCAL OSTEOMYELITIS IN CHILDREN: CAN IT BE TREATED WITH ORAL ANTIBIOTICS?
}

E Tsekoura, M.D., Ph.D.

Paediatric Department, Asklepieion General Hospital Athens Greece

\section{Background}

Acute osteomyelitis in children has an increased incidence over the last years of eight cases per 100.000 children per year with a high prevalence at 5 years of age. It occurs mainly in males possibly due to microtrauma and increased physical activity. Traditional treatment is based on prolonged intravenous antibiotics although recent reports suggest shorter intravenous courses and then switching to oral.

\section{Purpose}

We present two cases of staphylococcal osteomyelitis in order to discuss the use of oral antibiotics under certain conditions.

\section{Literature}

Bone and Joint Infections

The Pediatric Infectious Disease Journal: August 2017 - Volume 36 - Issue 8 - p 788-799

Evaluation of the current use of imaging modalities and pathogen detection in children with acute osteomyelitis and septic arthritis. Eur J Pediatr. 2018 May 4

\section{Bone and Joint Infections in Children: Acute} Hematogenous Osteomyelitis.

Indian J Pediatr. 2016 Aug;83(8):817-24.

Paediatric bone and joint infection. Iliadis $A D$, Ramachandran M. EFORT Open Rev. 2017 Mar $\frac{13 ; 2(1): 7-12 .}{2}$
Case 1: A 15 year old male adolescent presented with right hip pain and limp. In 48 hours he deteriorated with pyrexia $37.8^{\circ} \mathrm{C}$ and increased pain. Lab tests revealed increased neutrophile count, $C R P=189 \mathrm{mg} / \mathrm{L}, \quad E S R=52$. Hip ultrasound was normal. Patient refused admission and he was sent home with oral Amoxycillinclavulanate. Blood cultures taken on initial examination were MSSA positive and patient was called back to hospital. He was afebrile, painless and in good condition. MRI revealed osseous oedema of the right iliac bone, periosteal reaction and soft tissue muscular oedema (Fig 1). Because of these findings and despite his clinical and laboratory tests (CRP and ESR) improvement he received a sort course of 5 days i.v. antistaphylococcal penicillin which was switched to oral Amoxicillinclavulanate. Patient was reffered to paediatric orhtopeadic surgeon and received in total 8 weeks oral antibiotics. Repeated MRI 6 months later was normal (Fig.2)

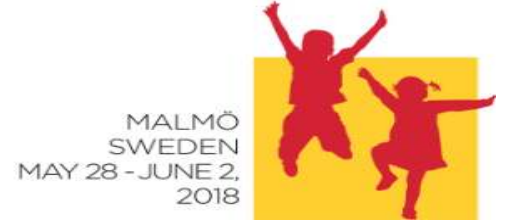

Case 2: A 81/2 year old boy admitted with a 48 hours history of fever $39^{\circ} \mathrm{C}$ with shivering, right groin pain and limp. There was a recent history of abdominal trauma. Initial orthopaedic examination diagnosed transient hip synovitis and he was treated with bed rest. CRP was 52.3 $\mathrm{mg} / \mathrm{L}, \quad E S R=50 \mathrm{mmHg}$. On D3 he was afebrile and free of symptoms. Nevertheless blood cultures taken on admition were MSSA positive. Subsequent MRI showed osseous oedema of the right trochaderic region with regional muscular oedema Because of MRI findings and despite his clinical ina lab improvement he was treated with a 5 days course of iv antistaphylococcal penicillin and he was switched to oral antibiotics for further treatment.

Discussion

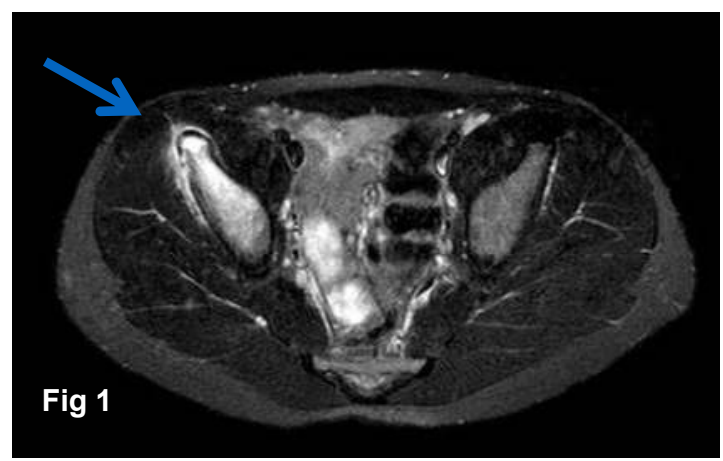

Although both patients had been improved with oral antibiotics on case1 or no antibiotics and bed rest on case2, because of subsequent report of positive MSSA cultures and MRI findings intravenous antibiotics were started afterwards, for a sort course and then switched to oral again. Course of the disease in both cases despite the delayed onset of intravenous antibiotics was unremarkable

Literature data support the use of oral antibiotics in benign peadiatric osteomyelitis cases. Further studies are required to identify optimum treatment algorithm.

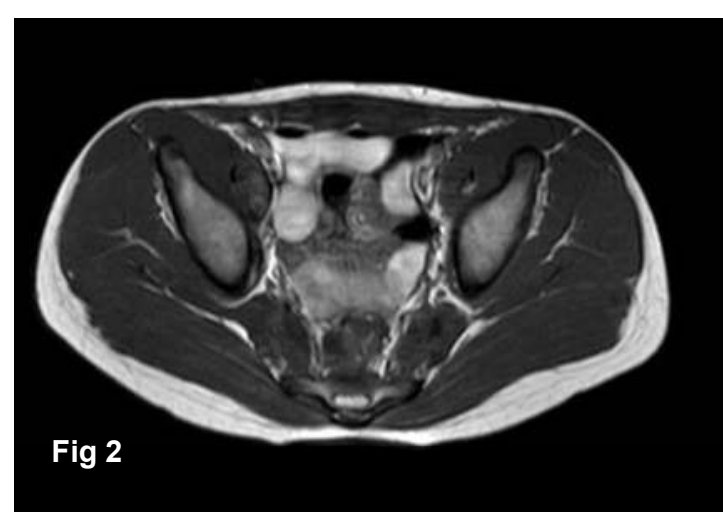

